\title{
On the Deceiving Aspects of Mixing Diagrams of Deep Cumulus Convection
}

\author{
STEVEn J. BÖIng, HARM J. J. JONKER, AND WiteK A. NAWARA \\ Delft University of Technology, Delft, Netherlands \\ A. PIER SiEBESMA \\ Delft University of Technology, Delft, and Royal Netherlands Meteorological Institute (KNMI), De Bilt, Netherlands
}

(Manuscript received 24 April 2013, in final form 20 August 2013)

\begin{abstract}
Mixing processes in deep precipitating cumulus clouds are investigated by tracking Lagrangian particles in a large-eddy simulation. The trajectories of particles are reconstructed and the thermodynamic properties of cloud air are studied using mixing diagrams. The trajectory analysis shows that the in-cloud mixing is entirely dominated by lateral entrainment and that there is no significant vertical mixing by downdrafts originating from cloud top. Yet the thermodynamic properties of the particles are located close to a line in the mixing diagrams, which appears to be consistent with two-point vertical mixing. An attempt is made to resolve this paradox using the buoyancy-sorting model of Taylor and Baker, but it is found that this model does not provide a full explanation for the location of particles in the mixing diagram. However, it is shown that the mixing-line behavior can be well understood from a simple analytically solvable model that uses a range of different lateral entrainment rates. Two further factors that determine the location of particles in the mixing diagram are identified: the removal of noncloudy air and precipitation effects. Finally, a thermodynamic argument is given that explains the absence of coherent downdrafts descending from cloud top.
\end{abstract}

\section{Introduction}

The transport of heat and moisture by convective clouds is one of the main uncertainties that affects the performance of weather and climate models. As these models often have a resolution that is too coarse to capture individual convective clouds, a statistical description or parameterization of cumulus convection is needed. One of the factors determining the transport of heat and moisture in a parameterized model is the entry level of environmental air into the updrafts and downdrafts.

The mixing mechanism in cumulus convection remains a topic of debate even after several decades of research. The two competing conceptual models about this issue have their origins in the 1940s and 1950s. Stommel (1947) proposed a model in which environmental air is entrained into the cloud at each level as an updraft rises through the atmosphere. This lateral entrainment model predicts that cumulus clouds have a lower total water content than the adiabatic value, and forms the basis of many operational

Corresponding author address: Steef Böing, TU Delft, P.O. Box 5048, 2600 GA Delft, Netherlands.

E-mail: s.j.boing@tudelft.nl weather prediction schemes. Squires (1958), however, argued that a model in which the mixing takes place between cloud base and cloud top gives a better explanation for the observed liquid water distributions in shallow cumulus convection. We will refer to Squires's model as cloud-top entrainment.

Further observational studies provided more evidence for cloud-top entrainment. Paluch (1979) used aircraft observations of cumulus clouds over Colorado to investigate the origin of cloud air. She made scatter diagrams using two variables that are (to good approximation) conserved during mixing processes. An example of such a mixing diagram is sketched in Fig. 1. The diagram is constructed with data from a large-eddy simulation (LES) of deep convection (see section 2). We will use moist static energy temperature [moist static energy divided by heat capacity of dry air: $\left.T_{h}=T+\left(g z+L_{v} q_{v}\right) / c_{p d}\right]$ and specific humidity $q_{t}$ as the conserved variables when we plot mixing diagrams. The $q_{t}$ axis is inverted so that cloud base properties appear at the bottom of the diagram. The thick line in the diagram represents the mean thermodynamic properties of the environment at different vertical levels. The collection of points shows the properties of cloudy parcels at a single level. 


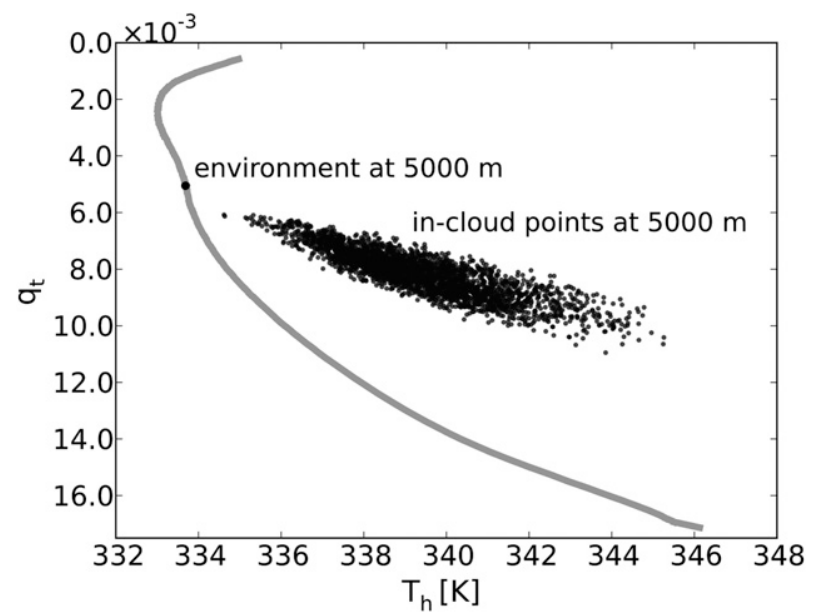

FIG. 1. A mixing diagram with the mean environmental profile shown as a thick line. The in-cloud points at $5 \mathrm{~km}$ are plotted as gray points.

Mixtures of air from two (but no more than two) different sources are located on a line in the mixing diagram. Paluch (1979) found that measurements in cumulus clouds were located on a mixing line that connects the environmental properties at cloud base and cloud top, and interpreted this as evidence for two-point mixing between cloud top and cloud base and, hence, for Squires's model. Later studies by, for example, Boatman and Auer (1983), LaMontagne and Telford (1983), Jensen et al. (1985), and Reuter and Yau (1987), confirmed the cloud-top mixing found by Paluch. A review article by Reuter (1986) summarizes the ideas in the middle of the 1980s.

Later studies criticized the conclusion that the mixing line indicates cloud-top entrainment [see also the recent review by de Rooy et al. (2012)]. These criticisms focused on two issues, as discussed below: the source levels of the mixing line and the interpretation given to the mixing line.

Several papers indicated that the mixing line had different source levels than Paluch found. Blyth et al. (1988) and Raga et al. (1990) found mixtures on a line that connects cloud base and a level close to observation level, rather than cloud base and cloud top. Whereas a mixing line that crosses cloud top implies cloud-top entrainment, a mixing line that crosses observation level indicates lateral entrainment. Malinowski and Pawłowska (1989) and Grabowski and Pawłowska (1993) showed biases in aircraft measurements can lead to an overestimation of the upper source level of entrained air.

Taylor and Baker (1991) provided an alternative interpretation of the mixing line, building on earlier work by Telford (1975). They proposed that the observed mixing line is a result of cloud parcels moving toward their level of neutral buoyancy. In their interpretation, positively buoyant parcels must have a source level below observation level, and negatively buoyant parcels have to come from above. Because of this buoyancy sorting process, the only part of the mixing diagram that is accessible for mixtures consists of two obtuse triangles that closely resemble a line. Besides buoyancy sorting, precipitation at any level in the cloud may also bias the interpretation of the mixing diagram, as Betts and Albrecht (1987) and Grabowski and Pawłowska (1993) pointed out. A third issue with the interpretation of the mixing line is that the variables in the mixing diagram are strongly self-correlated. As a result, observations will always have some tendency to fall on a line in the mixing diagram. This problem can be reduced by choosing a pair of variables in the mixing diagram that has a relatively low self-correlation (e.g., Heus et al. 2008).

With the advance of computer power, detailed studies of the flow in a cloud became possible. Lin and Arakawa (1997a) presented trajectories of Lagrangian particles from a cloud resolving model of deep convection. Lagrangian particles track the flow of air as it moves through the cloud. Lin and Arakawa did not find particles that originate from cloud top in the bulk of the cloud layer. However, these early simulations were two dimensional, and Lin and Arakawa presented results for only a small number of particles, which leaves the question whether the absence of downdrafts from cloud top is a representative result.

For shallow convection, Heus et al. (2008) showed unambiguously that cloud-top entrainment is insignificant. They presented statistics of a large number of particles using a three-dimensional model with detailed cloud dynamics, and found lateral entrainment to be dominant. However, Paluch's analysis concerned cumulus clouds that reach the upper troposphere, and downdrafts are generally thought to be more important in deeper convection.

This paper provides evidence that cloud-top entrainment is also insignificant in deep convection and that lateral entrainment is the dominant mixing mechanism (section 3). We use a three-dimensional LES and statistics of a large number of particles to support this point. However, we also find that the location of thermodynamic properties of the particles is close to a line in the mixing diagram (section 4), as is consistent with both aircraft observations and the numerical work of Lin and Arakawa (1997b) and Heus et al. (2008). Building on the work of Lin and Arakawa (1997b), we argue that observing mixing-line behavior does not necessarily imply two-point mixing. Using both an analytical framework and entraining parcel models, we show in section 5 how 
a mixing line can be easily reconciled with lateral mixing. The location of the in-cloud points in the diagram is also influenced by the removal of noncloudy air and precipitation effects. Finally, we present a thermodynamic argument that explains why downdrafts rarely descend farther than several hundred meters (section 6).

\section{Model description and case setup}

We use the Dutch Atmospheric Large-Eddy Simulation (DALES) model as described in Heus et al. (2010), with the modifications of Böing et al. (2012) for deep convection, such as the use of an anelastic approximation to account for changes in density in the vertical. The prognostic variables are the liquid water potential temperature $\theta_{l}$ and specific humidity $q_{t}$. For the construction of the mixing diagrams we use moist static energy temperature $T_{h}$. Although this is not a prognostic variable in the model, the advantage of this variable is that it is conserved when precipitation takes place.

The case is inspired by the Large-Scale BiosphereAtmosphere Experiment (LBA) intercomparison case described in (Grabowski et al. 2006). We use an idealized version of this case described in Wu et al. (2009), where we take the case with the most intense convection in their sensitivity study (U90). In this setup, radiative cooling and mean vertical motion are not taken into account. The initial profiles of relative humidity and virtual potential temperature lapse rate are shown in Fig. 2a. The relative humidity is $85 \%$ in the boundary layer, $90 \%$ in the lower troposphere, and decreases linearly above $6 \mathrm{~km}$. We prescribe the surface sensible and latent heat fluxes (161 and $343 \mathrm{~W} \mathrm{~m}^{-2}$, respectively). The domain has a horizontal grid spacing of $80 \mathrm{~m}$ and $1024 \times 1024$ points in the horizontal. Bryan et al. (2003) suggest that a grid spacing of the order of $100 \mathrm{~m}$ is needed to resolve deep cumulus convection, although inevitably part of the mixing processes between the clouds and their environment will take place on subgrid scales. The vertical grid spacing is $60 \mathrm{~m}$ with 400 levels. We run the model for $10 \mathrm{~h}$ of simulation time, and focus on particle statistics sampled after $8 \mathrm{~h}$. Figure $2 \mathrm{~b}$ shows a snapshot of the cloud condensate path in the domain after $8 \mathrm{~h}$. The cold pools start to grow toward the scale of the domain, and larger domains would be recommended for longer simulations.

The Lagrangian particle routine is described by Heus et al. (2008). We release 15 million particles that are initially distributed homogeneously throughout the domain, with a concentration proportional to atmospheric density. We record their position and the corresponding thermodynamic properties every $20 \mathrm{~s}$ (the time integration step is smaller).
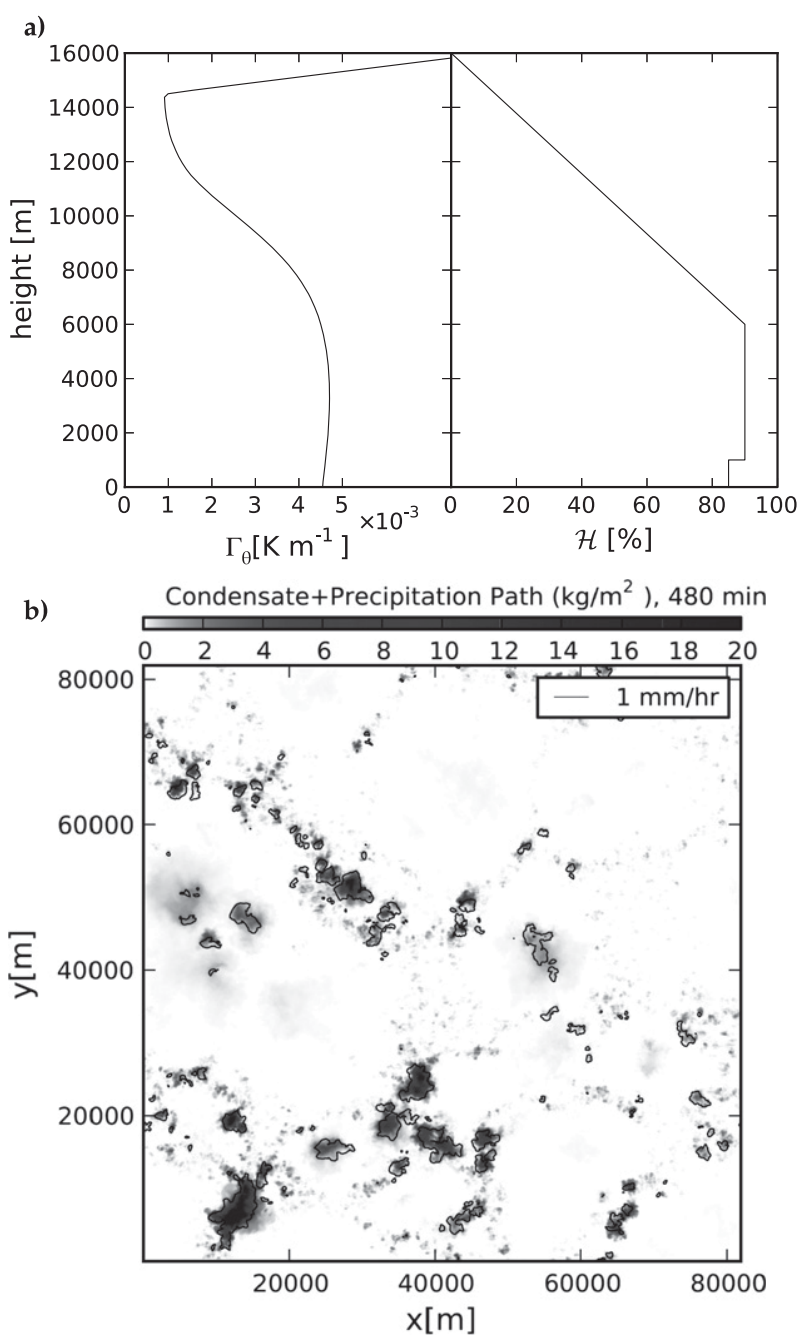

FIG. 2. (a) Initial profiles of potential temperature lapse rate and relative humidity. (b) Snapshot of cloud condensate path and surface precipitation rate after $8 \mathrm{~h}$.

Particle statistics are constructed from snapshots of the cloud field: we consider all particles that are located inside the cloud at some point in time (observation time). As we have both forward and the backward information on the trajectories of the particles, we can trace the particles back to the last time that they entered the cloud (entry time) and forward to when they leave the cloud (exit time). This means particles stay in a single cloud. We will use the entry level to investigate where the air in the clouds comes from.

\section{Trajectories of particles in the clouds}

In this section we address the question where the air in deep cumulus clouds originates. Figure 3 presents a sketch of a histogram [following Heus et al. (2008)], where 


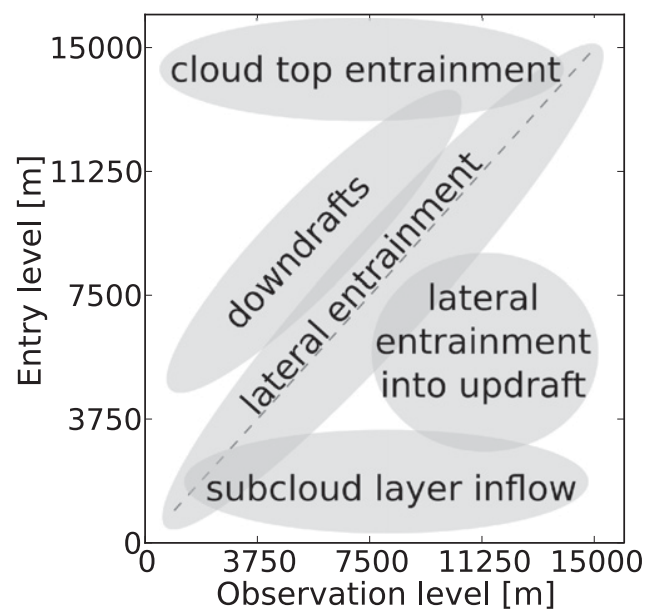

FIG. 3. Conceptual plot indicating the position of subcloud-layer inflow, lateral entrainment, downdrafts, and cloud-top entrainment in the entry-observation histogram.

observation and entry level of particles are plotted on the axes. The sketch shows the interpretation of the various locations in the histogram. The diagonal line indicates equal observation and entry levels; particles that lie above this line come from above. Particles traveling downward from cloud top are found at the top of the diagram, whereas inflow at cloud base is found at the bottom. Lateral entrainment shows up near the diagonal (if the particles stay at the level of entry) or below it (if the particles travel upward). In Fig. 4a, we present a histogram from the LES simulation. We consider all particles (about 360000 ) that are in the clouds (i.e., where $q_{l}>0$ ) after $480 \mathrm{~min}$ of simulation time. The shade represents the number of particles that have the corresponding combination of entry and observation level. We find that most particles enter the cloud layer either through the subcloud layer or close to their observation level. This bimodal distribution of entry level is remarkably similar to the results of Heus et al. (2008) for shallow convection. Some downdrafts occur near cloud top, but they do not descend over large distances.

A one-dimensional histogram of the entry level at a single observation level in the middle of the cloud layer is shown in Fig. 4b. Many particles originate either from the subcloud layer or from a level close to observation level. However, some particles have their entry levels between cloud base and observation height. These particles are entrained laterally and subsequently transported upward by the cloud. Very few particles originate from levels above observation height.

The conclusion is clear: lateral entrainment is the predominant form of mixing in deep clouds. There is no substantial vertical mixing by downdrafts in the clouds. a)

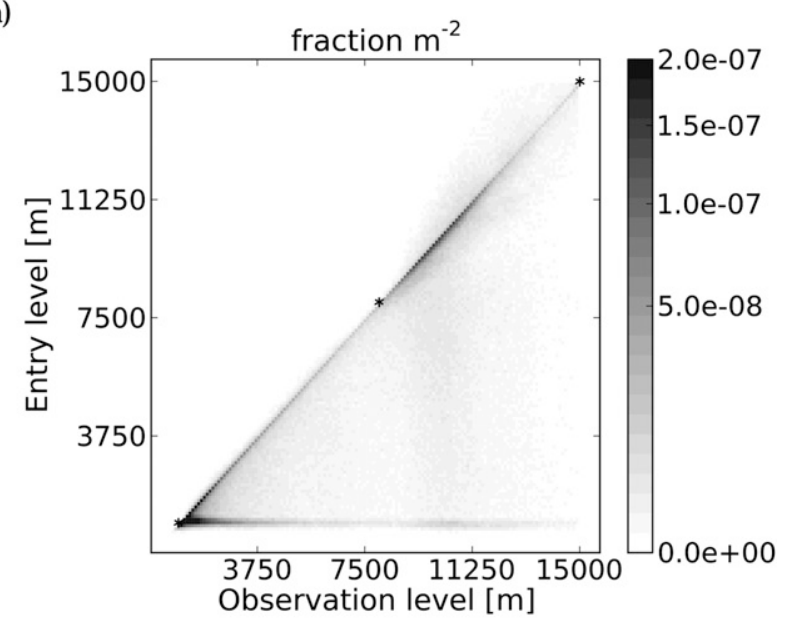

b)

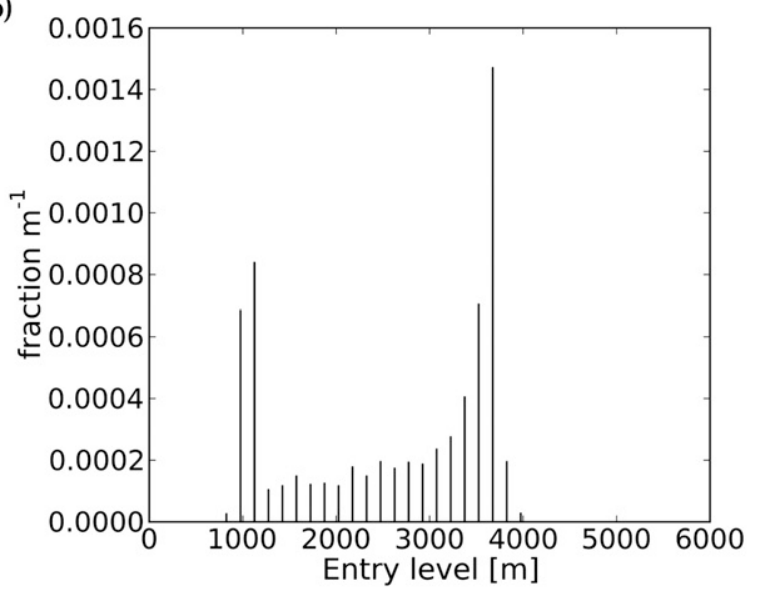

c)

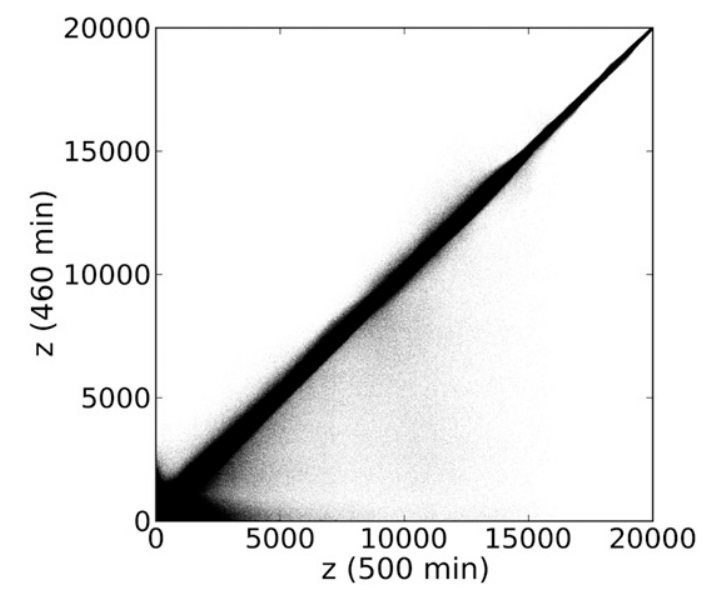

FIG. 4. (a) Histogram of entry and observation level; markers indicate the diagonal. (b) One-dimensional histogram of entry level for observation levels between 3625 and $3725 \mathrm{~m}$. (c) Scatterplot of all particle heights $20 \mathrm{~min}$ before and after observation time (480 min). 


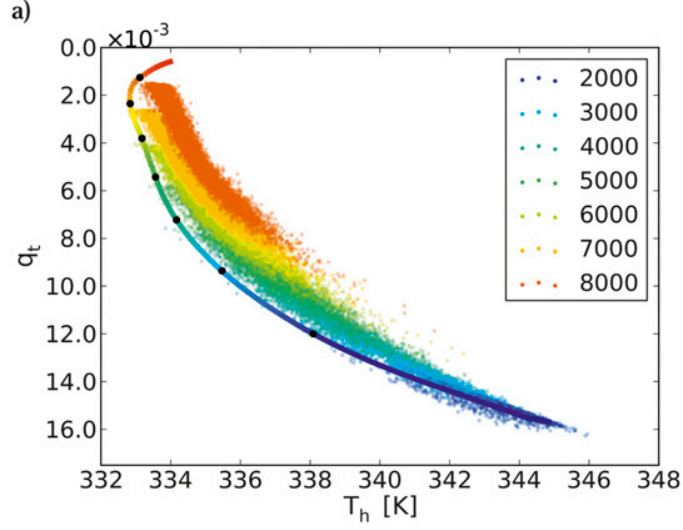

c)

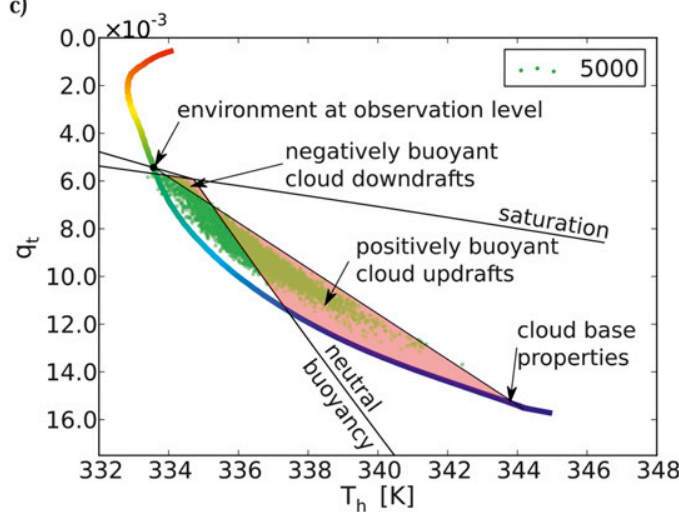

b)

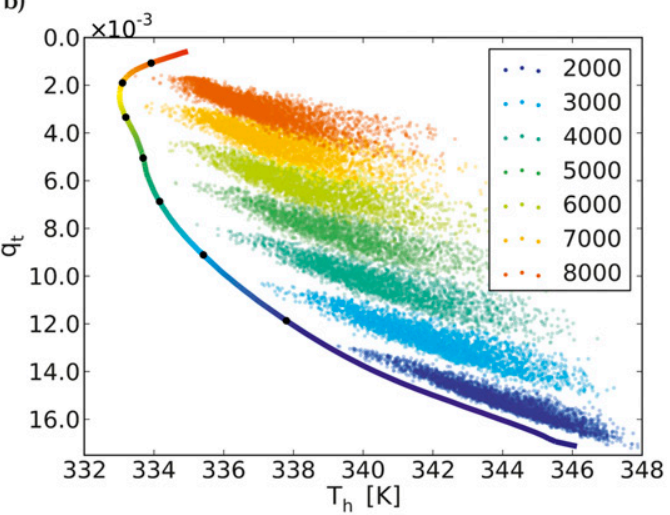

d)

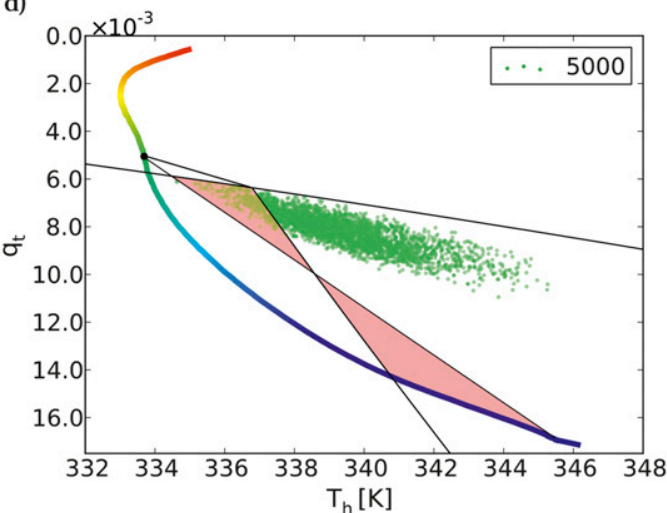

FIG. 5. Mixing diagrams that display in-cloud particles and the mean environment. Color is used to indicate height. The in-cloud points are colored with the same shades as the environmental curve: (a) without precipitation and (b) with precipitation. Areas accessible according to Taylor and Baker (1991; see main text) for a single level:

(c) without precipitation and (d) with precipitation. A random selection of 10000 particles is used.

Figure $4 \mathrm{c}$ shows a scatterplot of the location of all 15 million particles $20 \mathrm{~min}$ before and after observation time. Here, it is seen that particles descending over large vertical distances are also absent outside the clouds.

The present study is concerned only with the net movement of particles as they move in the cloud. Yeo and Romps (2013) found particles that enter the cloud near its top in a study of a single cloud, which is consistent with our findings. Yet in the present framework, if a particle enters the cloud near its top but does not descend afterward, this is termed "lateral entrainment at cloud top" rather than "cloud-top entrainment."

\section{Mixing diagrams of the large-eddy simulation}

In the previous section we showed that Lagrangian particles enter the cloud either through the subcloud layer or because of lateral entrainment. Mixing diagrams tell us not only about the origin of cloud air but also about what happens to particles as they rise or descend in the cloud. For example, particles corresponding to an undilute updraft stay at the same position in the mixing diagram, whereas mixing causes particles to move toward each other in the diagram. In the previous section, we found lateral mixing from many levels rather than mixing between two levels. We would therefore intuitively expect a mixing diagram that does not look like a line. As the location of points in the mixing diagram may be affected by precipitation, we first consider the same LES case, but intentionally switch off precipitation. Figure 5a shows the mixing diagram of the particles after $480 \mathrm{~min}$. The colored dots indicate the thermodynamic properties of the in-cloud particles. The particle properties are plotted every $1000 \mathrm{~m}$, and each color in the plot corresponds to a different height (heights are measured with respect to the ground level). The colors are the same as those used for the environmental curve.

The cloud properties are located in a small region that runs parallel to a segment of the environmental curve between cloud base and observation level, and resembles two-point mixing. The in-cloud points do not retain cloud base properties, with the exception of a few points 
in the lowest two levels. This indicates entrainment of air from levels between cloud base and observation level.

Figure $5 \mathrm{~b}$ shows the mixing diagram for the precipitating case discussed in the previous section. The in-cloud points are located farther away from the environmental curve. The parcels that have experienced relatively little mixing (those with highest $T_{h}$ ) have shifted upward in the diagram. This is because they have high liquid water content, and will lose water because of precipitation. Some of the particles have properties close to those of the environment near the level of observation.

To reconcile the observed mixing diagrams with a lateral entrainment mechanism, we return to the hypothesis of Taylor and Baker (1991), who proposed that only certain areas in the mixing diagram are accessible. We plot these areas for a single level in Figs. 5c and 5d. The lower triangle corresponds to positively buoyant cloud updrafts. These must have properties that are a mixture of the sources below observation level; therefore, the corresponding points must lie below the line that connects cloud-base properties to observation-level properties of the environment. The upper triangle corresponds to negatively buoyant mixtures that are a mixture of cloud-base air and air with a source level above observation level. Note that this analysis has some limitations: for example, it does not consider mixtures between updrafts and downdrafts.

It appears that the model of Taylor and Baker cannot fully constrain the location of the in-cloud points: in large parts of the red areas, no particles are found. Also, some of the particles are found outside the red areas. By looking at scatter diagrams of vertical velocity and buoyancy (not shown), we found that there are negatively buoyant ascending particles (overshoots). Although the model of Taylor and Baker was designed for nonprecipitating cases, we also consider the location of particles in the precipitating case. These particles are found mostly to the right of the areas allowed by the model of Taylor and Baker, rather than to their left. Since precipitation removes water, updrafts can now have properties that lie above the line that connects cloud base to observation level, even in the absence of downdrafts.

\section{Apparent two-point mixing explained}

To better understand the mixing diagram behavior, we explore below a few simple entraining parcel models. We will talk about parcels rather than particles in this section in order to distinguish the parcel model results from those of the Lagrangian particles. This terminology is also consistent with previous work on the subject. We argue that a successful parcel model needs to have three ingredients: differences in entrainment rate, removal of noncloudy air from the updraft, and precipitation. Models that have these three ingredients produce mixing diagrams comparable to those in the LES.

\section{a. An idealized parcel model}

Can we understand how differences in entrainment rate constrain the location of in-cloud points in the mixing diagram? An idealized system of equations that has analytical solutions illustrates how this happens. The system is an abstraction of an entraining-parcel model: we will look at a parcel model that can be directly compared with the LES in section $5 \mathrm{~b}$.

The environmental profile in the mixing diagram has the important property that it is curved. This property also underlies Paluch's analysis: if the environmental curve were a straight line in the mixing diagram, any mixture would lie on this line, even it has more than two source levels. A local minimum in moist static energy is a typical feature of tropical deep convective environments (e.g., Emanuel 1994). However, a similar minimum can also be found for shallow convection cases by choosing an appropriate basis to span the thermodynamic coordinates (Heus et al. 2008). The existence of such a minimum is exploited in the current analysis. To capture the relevant behavior, we use a simple model of a parcel that entrains environmental properties $T_{e}$ and $Q_{e}$, which can be thought of as a nondimensional moist static energy temperature and specific humidity:

$$
\begin{aligned}
\frac{d}{d z} T_{p}=-\epsilon\left(T_{p}-T_{e}\right) & T_{p}(0)=T_{e}(0) \quad \text { and } \\
\frac{d}{d z} Q_{p}=-\epsilon\left(Q_{p}-Q_{e}\right) & Q_{p}(0)=Q_{e}(0) .
\end{aligned}
$$

Here, $T_{p}$ and $Q_{p}$ are parcel properties, and $\epsilon$ is a nondimensional parcel entrainment rate. There are no sinks of $Q$ and $T$ in this model. We consider a nondimensional height $z$ between 0 and 1 , where $z=0$ corresponds to cloud base. The environmental properties lie on a parabola in the $Q-T$ phase space and are given by $T_{e}=$ $(2 z-1)^{2}$ and $Q_{e}=1-z$. The parcels have no initial excess in the properties at cloud base. The properties at higher levels can be solved analytically:

$$
\begin{gathered}
Q_{p}(\epsilon, z)=-\frac{\epsilon z+e^{-\epsilon z}-\epsilon-1}{\epsilon} \text { and } \\
T_{p}(\epsilon, z) \\
=\frac{\epsilon^{2}+4 \epsilon+8-4 \epsilon^{2} z+4 z^{2} \epsilon^{2}-4 \epsilon e^{-\epsilon z}-8 e^{-\epsilon z}-8 \epsilon z}{\epsilon^{2}} .
\end{gathered}
$$


We have plotted several solutions to this system with different entrainment rates in Fig. 6a. The $Q$ axis is inverted, as in the LES mixing diagrams. The dark lines indicate phase-space trajectories corresponding to different values of $\epsilon$.

Figure $6 \mathrm{~b}$ shows the analytical solution for the properties of a range of parcels with different $\epsilon$ but at the same height. It is crucial that we are not looking at the behavior of a single parcel, but consider a single height, as in an airplane transect. Solutions are plotted at dimensionless heights $z=0.5$ and $z=0.9$. The differences in entrainment between parcels result in mixing-line behavior near cloud base. Nearly undilute parcels, which would keep their cloud-base properties, were not observed in the LES (Fig. 5a). We therefore expect very low entrainment rates not to occur in the parcel ensemble. Very high entrainment rates will also be excluded, as parcels with a high entrainment rate do not stay cloudy in the LES. The darker-colored part of the solution has a (dimensionless) entrainment rate between 0.4 and 4.

Can we explain the quasi-linear behavior of the particle clusters in Fig. 5a? We derive the slope of the mixing line near cloud base by making a first-order Taylor expansion of $T_{p}$ and $Q_{p}$ in terms of $\epsilon$. The Taylor expansions can be combined into an equation for $Q_{p}$ in terms of $T_{p}$ and $z$. The entrainment rate has been expressed in terms of the other variables, and $Q_{p}$ is used as an independent variable here. In this manner, the equation gives an expression for the mixing line at each level as it appears in the mixing diagram:

$$
T_{p}=-3+\frac{8}{3} z+Q_{p}\left(4-\frac{8}{3} z\right) .
$$

Figure $6 \mathrm{~b}$ shows a dashed line that is the linear approximation given above for $z=0.9$ and a dotted line connecting cloud-base properties and environmental properties at $z=0.9$. These lines constrain the analytical solution of the mixing curve to an obtuse cone in the mixing diagram. The full analytical solution follows the linear approximation closely for parcels with small or moderate entrainment rates, suggesting that the linear approximation is a good first approximation of the mixing line. No parcels are located below the linear approximation.

Does the occurrence of a mixing line depend on the choice of an entrainment rate that is constant with height? If we take an entrainment rate that is proportional to the inverse of height $\left(\epsilon=\epsilon_{0} / z\right.$-an extreme case where entrainment is very high near cloud base but excesses develop at higher levels), we can once again find analytical solutions for $Q_{p}$ and $T_{p}$. In this case, it is a)

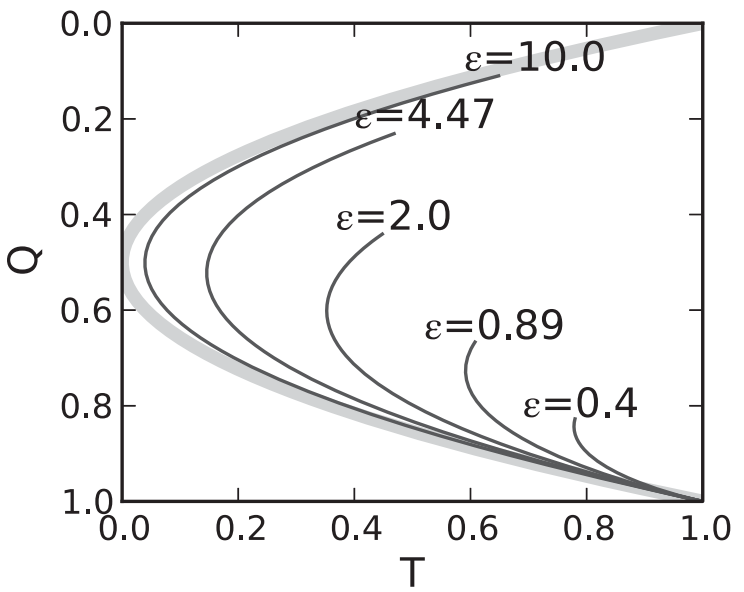

b)

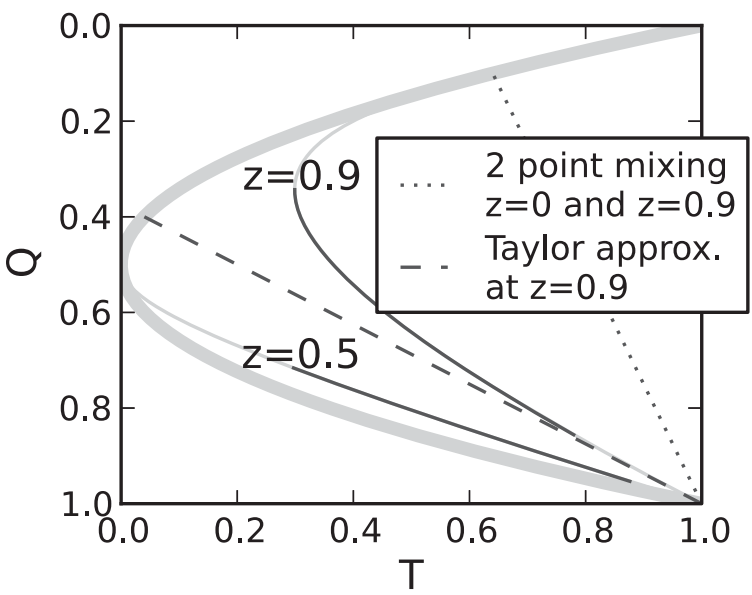

c)

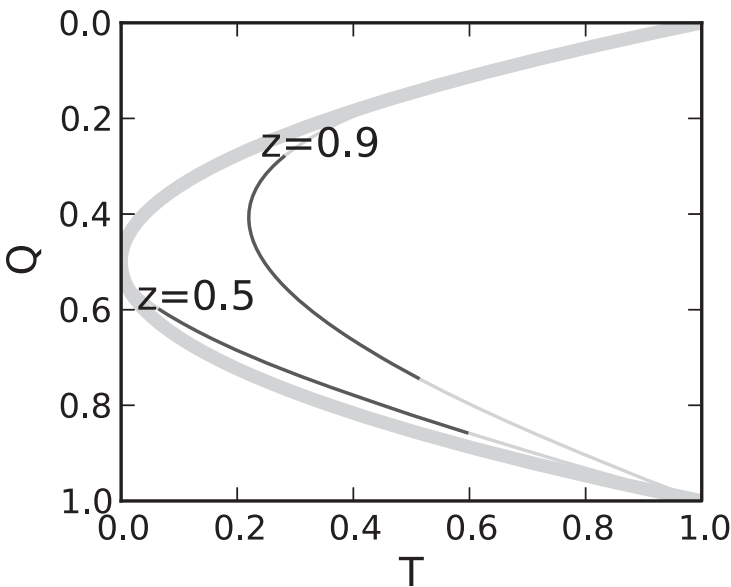

FIG. 6. Solutions to the simple system of equations that is used to study entrainment in section 5a. (a) Trajectories for a single entrainment rate. (b) The analytical solutions of the parcel properties at a single height fall between two lines: the dotted line connecting cloud-base properties to environmental properties and the Taylor approximation (dashed) in Eq. (5). The darker-colored part of the solution has an entrainment rate between 0.4 and 4. (c) As in (b), except using an entrainment rate $\epsilon_{0} / z$, where the darker part has $\epsilon_{0}$ between 0.4 and 4 . 
even possible to find an analytical formulation of $T_{p}$ in terms of $Q_{p}$ and $z$ (as in the previous Taylor expansion):

$$
T_{p}=\frac{3-6 z+4 z^{2}-7 Q_{p}+4 Q_{p}^{2}+8 Q_{p} z-4 Q_{p} z^{2}}{Q_{p}-1+2 z} .
$$

This solution is plotted for several values of $z$ in Fig. $6 \mathrm{c}$ (with the constraint $Q_{p}>Q_{e}$, which corresponds to positive entrainment rates). Again, we observe that the solutions at a constant height resemble two-point mixing, although the solutions remain closer to the environmental curve than in Fig. 6b. This shows that the occurrence of a mixing line is not very sensitive to the entrainment formulation.

\section{b. A parcel model for deep convection}

Building on the work of Lin and Arakawa (1997b), we now consider a parcel model that is much closer to the LES. This parcel model is governed by a similar set of equations as the analytical model in the previous section:

$$
\begin{aligned}
& \frac{d}{d z} T_{h, p}=-\epsilon\left(T_{h, p}-T_{h, e}\right) \quad T_{h, p}\left(z_{\mathrm{cb}}\right)=T_{h, 0} \quad \text { and } \\
& \frac{d}{d z} q_{t, p}=-\epsilon\left(q_{t, p}-q_{t, e}\right) \quad q_{t, p}\left(z_{\mathrm{cb}}\right)=q_{t, 0}
\end{aligned}
$$

Here, $z_{\mathrm{cb}}$ is the height of the cloud base. The parcel model uses moist static energy as a prognostic variable, rather than a diagnostic one. The calculation of saturation specific humidity is the same as in DALES. The parcel is initialized with a moisture excess of $1 \mathrm{~g} \mathrm{~kg}^{-1}$ and a temperature deficit of $0.5 \mathrm{~K}$ at cloud base in order for the parcel to be cloudy at this level. The model is not very sensitive to these excesses, as long as they remain small. The environmental profiles are taken as the precipitating LES case horizontal mean $q_{t}$ and $T_{h}$ profiles after $8 \mathrm{~h}$. The easiest method of bringing differences into the entrainment is by giving each parcel a different $\epsilon$ that remains constant with height, as in the previous section. The differences in $\epsilon$ may be due to, for instance, the particles being at different locations in a cloud (Dawe and Austin 2011).

We study an ensemble of parcels with $\epsilon$ between $1.5 \times$ $10^{-4}$ and $1.0 \times 10^{-3} \mathrm{~m}^{-1}$. The minimum entrainment rate is chosen such that the location where the mixing line starts is comparable with that in the LES results. The choice of maximum entrainment rate is less important, as the model also removes unsaturated parcels from the ensemble.

Since unsaturated parcels are removed, parcels that entrain strongly will not reach a location close to the environmental curve in the mixing diagram. Figure $7 \mathrm{a}$ shows the results of the parcel model without precipitation. The location of the mixing line is similar to that in the LES case without precipitation (Fig. 5a), especially for the lower levels. The model predicts slightly too high moisture content as compared to the LES. The parcel model does not produce any scatter of parcel properties perpendicular to the mixing line.

\section{c. Precipitation}

The effect of precipitation is parameterized in the parcel model by removing condensate and cloud ice in excess of a certain threshold $q_{f}=1 \mathrm{~g} \mathrm{~kg}^{-1}$ over a length scale $\ell_{f}=1000 \mathrm{~m}$ [which is similar to Lin and Arakawa (1997b); this is in effect a Kessler scheme where the time scale has been replaced by a length scale]. The corresponding parcel equations read

$$
\begin{aligned}
\frac{d}{d z} T_{h, p} & =-\epsilon\left(T_{h, p}-T_{h, e}\right), \\
\frac{d}{d z} q_{t, p} & =-\epsilon\left(q_{t, p}-q_{t, e}\right)+S_{q}, \quad \text { and } \\
S_{q} & =\min \left(-\frac{q_{l, p}+q_{i, p}-q_{f}}{\ell_{f}}, 0\right) .
\end{aligned}
$$

This approach to microphysics is rather crude, but serves to illustrate the differences between a precipitating and a nonprecipitating case. In particular, when we compare the location of the mixing line at different heights (as shown in Fig. 7b) with the nonprecipitating case (Fig. 7a), the mixing lines are farther separated in the diagram for the precipitating case. The slope of the mixing line also changes because the parcels with the highest liquid water content will experience most precipitation. The location of the mixing lines is now in better agreement with the precipitating LES (Fig. 5b).

\section{d. A stochastic mixing model}

In the previous paragraphs, we looked into a parcel model where $\epsilon$ was constant with height for each parcel, but could vary between parcels. Neggers et al. (2002) presented a parcel model where the entrainment rate depends on vertical velocity, which is also able to represent mixing-line behavior. In this model, variability is introduced by starting the parcels with different vertical velocities at cloud base. In both the model of Lin and Arakawa and that of Neggers et al., a lower entrainment rate just above cloud base corresponds to a lower entrainment rate throughout the rest of the troposphere.

The cloud thermodynamic properties given by the model in the previous section are constrained to a line, 


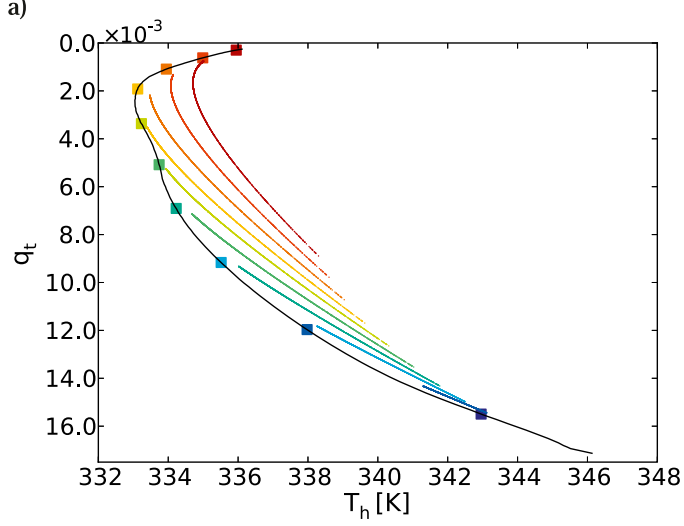

c)

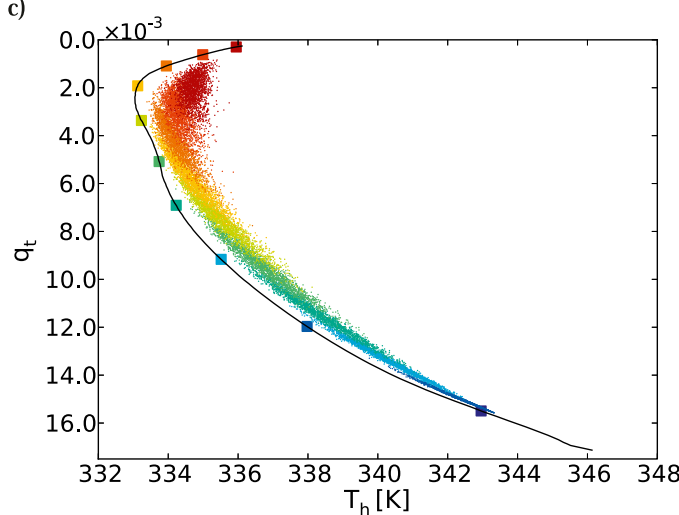

b)

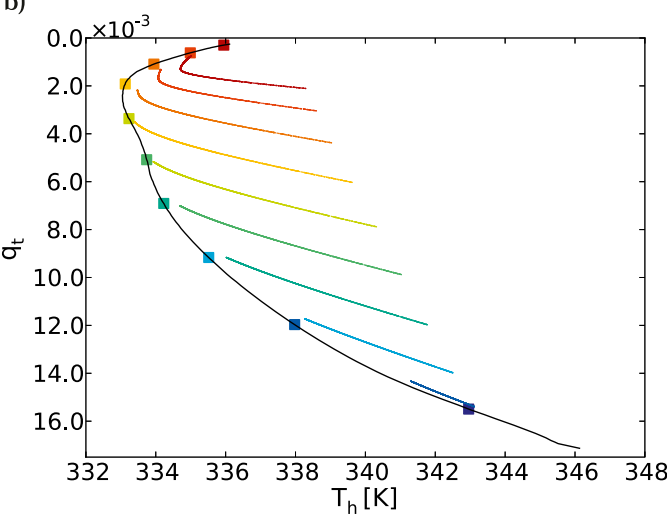

d)

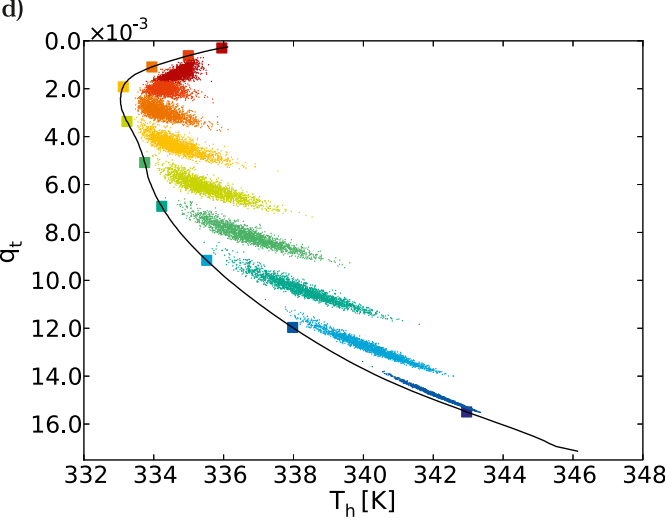

FIG. 7. Mixing diagrams corresponding to the stochastic parcel model, where the environmental profile is taken from the simulation of deep convection. (a) A mixing rate $\epsilon$ that is constant with height and differs for each parcel; (b) as in (a), but with a simple precipitation model, as described in the main text; (c) the stochastic mixing model; and

(d) the stochastic mixing model with precipitation.

whereas the LES shows more scatter of the in-cloud properties. For shallow convection, Romps and Kuang (2010) proposed a model that considers entrainment to be a stochastic process, where the entrainment at each height is fully independent of the entrainment rate at lower heights. This approach has recently been implemented in a single-column model by Sušelj et al. (2013). In this section, we argue that such a model, which we call a stochastic mixing model, can give a better representation of the variability of cloud properties in the LES data.

In our stochastic mixing model, the entrainment rate in Eqs. (9)-(11) is drawn from a probability density function at each level. This is somewhat different from the model of Romps and Kuang, where entrainment takes place only at certain heights, and the distance to the next entrainment event is drawn from a probability distribution as well as the entrainment rate.

We draw $\epsilon$ at each level from a gamma distribution $\Gamma(\epsilon ; \lambda \Delta z, \mu / \Delta z)$, where $\Delta z$ is the parcel model grid spacing, $\lambda \Delta z$ the so-called shape parameter, and $\mu / \Delta z$ the scale parameter. Note that $\Gamma$ denotes the gamma distribution and not the gamma function. The choice for a gamma distribution originated not so much from physical arguments, but rather from convenience arguments: we need a distribution that is guaranteed to yield only positive $\epsilon$ values (which immediately rules out, e.g., Gaussian distributions), and we require the model results to be grid-spacing independent. As shown in the appendix, the gamma distribution satisfies all these requirements because of proper scaling. The mean entrainment rate and the variance of entrainment rate can be independently set with the parameters of the distribution $\lambda$ and $\mu$ (appendix). The model does not account for any correlation in entrainment between vertical levels that are close; in this respect the full correlation model discussed in the previous section represents the other extreme.

Figures $7 \mathrm{c}$ and $7 \mathrm{~d}$ show the mixing diagrams for parameters $\lambda=0.002 \mathrm{~m}^{-1}$ and $\mu=0.12$, both with and without precipitation. In this case, the ensemble-mean entrainment rate $\bar{\epsilon}=\lambda \mu=2.4 \times 10^{-4} \mathrm{~m}^{-1}$ and the standard deviation 
of the vertical-mean entrainment rate $\sigma_{\langle\epsilon\rangle}$ over a $1000-\mathrm{m}$ interval is approximately $1.8 \times 10^{-4} \mathrm{~m}^{-1}$. This mean and standard deviation have been chosen such that the parcel model reproduces a similar spread in cloud properties as the LES. The behavior is similar to that of the model in the previous section, but, like the LES data, the parcels are no longer confined to a single curve. This spread reflects a difference in the entrainment history of the particles. The similarities between the parcel models are more striking than their differences-remember that the parcel models represent entrainment in very different ways.

The stochastic mixing model is the most complex parcel model that we consider. As particles never descend over large distances in the cloud, a model with only updrafts captures the first-order behavior of the mixing diagram. To improve on the current model, it could be extended by introducing a vertical velocity equation and allowing parcels to overshoot their level of neutral buoyancy, continue mixing, and return to their level of neutral buoyancy. The behavior of mixing in overshoots is discussed by Blyth and Cooper (1988), Blyth et al. (1988), and Carpenter et al. (1998). In the parcel model, we would need to make assumptions on the mixing between downdrafts and their environment. To get an idea of the behavior of negatively buoyant cloud parcels, we can also apply a more generic argument, as is shown in the next section.

\section{Thermodynamic constraints on downdrafts}

We found that the mixing-diagram behavior is consistent with the absence of coherent in-cloud downdrafts that descend over more than a few hundred meters. But why are such downdrafts unlikely? Below, we provide a thermodynamic argument that explains the absence of downdrafts that transport air over larger distances. We assume the downdrafts descend to their level of neutral buoyancy.

The argument is based on a comparison of mixingdiagram behavior at two levels. At each level, the mixing diagram is partitioned into four sections, based on the positive or negative parcel buoyancy and the absence or presence of liquid water. Analysis of the properties of the negatively buoyant in-cloud particles from the LES at the upper level subsequently gives constraints on vertical descent. The negatively buoyant in-cloud particles at $5730 \mathrm{~m}$ (for the precipitating case) are indicated as dots in the light area in Fig. 8. The dark area is the cloudy part of the diagram at $4710 \mathrm{~m}$. The two levels have been chosen so that there is no overlap between these areas, which means that if a negatively buoyant cloudy parcel descends from 5730 to $4710 \mathrm{~m}$ without mixing, it will not be cloudy anymore. Descents of a few

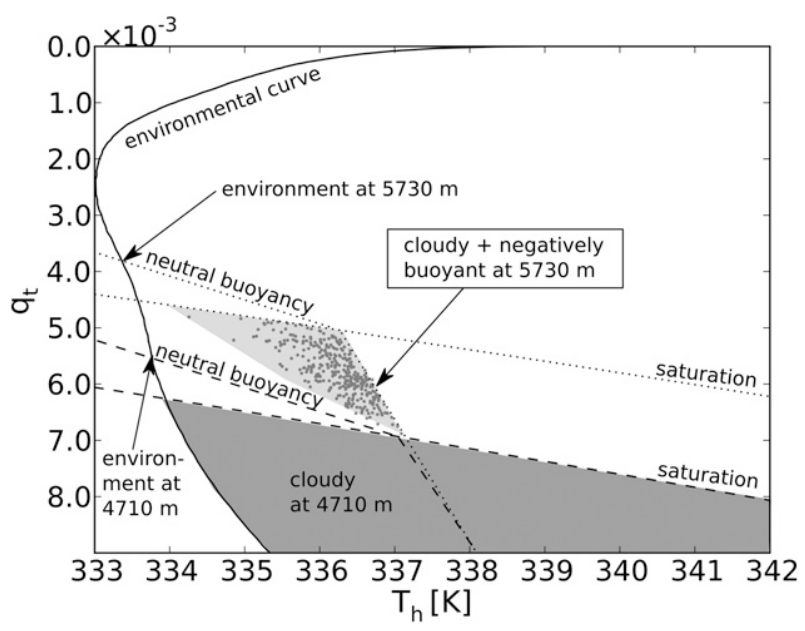

FIG. 8. Mixing diagram showing the neutral buoyancy and saturation curves at two different levels, as well as the environmental curve. The light shading corresponds to cloudy and negatively buoyant parcels in the LES at the upper level. All of the parcels in the LES (dots) would be unsaturated and positively buoyant at the lower level. The dark shading corresponds to cloudy conditions at the lower level.

hundred meters are in agreement with the magnitude of the largest descents in the LES.

Mixing with the environment does not help the descending parcels to retain their negative buoyancy, as the environmental curve between 4710 and $5730 \mathrm{~m}$ is also located above the 4710-m neutral-buoyancy line in the mixing diagram.

The downdraft loses its negative buoyancy as it descends over larger distances and becomes a dry parcel. Without mixing, its virtual temperature will follow a dry adiabatic lapse rate. This is in strong contrast to the behavior of undilute updrafts, which follow the moist adiabatic lapse rate and become more positively buoyant as they rise through the atmosphere. Mixing, which causes a parcel to dry at the higher levels, is responsible for this irreversible lapse rate. The analysis in the current work is similar to that of Betts (1973), who considered the asymmetry between updrafts and downdrafts in moist convection due to irreversible mixing from the perspective of a single parcel. Betts argued that in the updrafts, condensation due to lifting is partly compensated owing to evaporation by mixing, whereas in the downdrafts both descent and mixing cause evaporation.

The "lapse rate argument" presented above is consistent with the fact that downdrafts are able to descend much farther once they enter the subcloud layer. When the downdrafts reach the top of the subcloud layer, the environmental properties do not change strongly with height anymore (the subcloud layer is relatively well mixed), and the parcel can descend farther without impediments. 
There are two further effects that can contribute to the formation of downdrafts: 1) precipitation loading, which has a temporary effect on the buoyancy (precipitation eventually falls out), and 2) evaporation/ melting of precipitation. Evaporatively driven downdrafts may be more important in an environment with strong wind shear, where precipitation falls out of the cloud at higher levels or in environments with a lower relative humidity. The high relative humidity in the present setup is characteristic of the wet season over the Amazon as well as maritime conditions. Evaporation of precipitation occurs outside the cloud, so it does not play a role for in-cloud thermodynamic properties. Irrespective of the creation of downdrafts, evaporation of precipitation is an effective way of transporting moisture downward in the atmosphere. Melting is not taken into account here, but the energy absorbed during melting is much lower than that of evaporation.

\section{Conclusions and discussion}

We studied the mechanisms of mixing in deep, precipitating cumulus clouds with Lagrangian particle tracking. A large majority of particles are transported upward in the cloud. Histograms of the trajectories of particles in the clouds show that cloud-top entrainment does not occur in any significant way, even though convection reaches the upper troposphere.

Nevertheless, the mixing diagrams plotted with the Lagrangian particles show in-cloud properties that may, at first sight, suggest two-point mixing. An explanation for this behavior was found using parcel models. The combination of differences in lateral entrainment rate and the removal of unsaturated air explains the preferential location of cloud properties in the mixing diagram. Precipitation shifts this location toward lower specific humidity values. A model that incorporates these three effects captures the main characteristics of the mixing diagram. We found that two parcel models that represent lateral entrainment in very different ways are both suitable to represent the mixing-diagram behavior, with the stochastic mixing model better at capturing the scatter of in-cloud thermodynamic properties.

Using arguments similar to those presented by Betts (1973), we have also shown why downdrafts can travel only a limited vertical distance. We showed that negatively buoyant cloud air soon becomes positively buoyant and unsaturated as it descends.

Parcel models have the drawback that their parameters cannot be diagnosed directly from an LES in the same manner as the parameters in a bulk-plume framework. Nie and Kuang (2012) have recently explored a method to compare the way cloud properties change from one level to the next in a parcel model to LES results. Estimating the probability density functions of entrainment rate from aircraft observations is difficult, as an aircraft does not track individual parcels. Lu et al. (2012) presented observations of probability distributions functions of entrainment rate in shallow cumulus clouds as a function of distance to the cloud edge. The lognormal distributions of entrainment rate that they find are similar to the gamma distribution that was used in section 5 .

Does the fact that coherent downdrafts do not transport cloud air over large distances on the short time scale mean that downdrafts can be ignored in parameterizations? This question is beyond the scope of the current paper. Downdrafts at the edge of the cloud [as discussed in, e.g., Jonas (1990), Rodts et al. (2003), and Heus and Jonker (2008)] are known to influence transport of chemical species (e.g., Verzijlbergh et al. 2009), and downdrafts driven by precipitation of hydrometeors may become more important in sheared and dry environments. Precipitationdriven downdrafts are also crucial in the subcloud layer, if only because they play an important role in organizing the convection (e.g., Khairoutdinov and Randall 2006; Böing et al. 2012). It is also important to distinguish convective downdrafts from the large-scale compensating subsidence of the environment, which is needed to warm and dry the tropical atmosphere and adjust it toward radiative-convective equilibrium.

Acknowledgments. This work was sponsored by SURFSARA for the use of supercomputer facilities, with financial support of NWO. We thank three anonymous reviewers for their constructive feedback and Jerôme Schalkwijk, Stephan de Roode, and David Romps for earlier discussions on parts of this work.

\section{APPENDIX}

\section{Choice of the Entrainment Probability Distribution}

In section 5, we draw the entrainment rate from a gamma distribution. We choose this distribution because it ensures positive entrainment rates and makes the model independent of grid spacing (in the limit of small grid spacing), as discussed below. In a parcel model with vertical spacing $\Delta z$, the vertically integrated entrainment rate between two heights $z_{1}$ and $z_{2}$ is given by

$$
\mathcal{E}_{z_{1}, z_{2}}=\int_{z_{1}}^{z_{2}} \epsilon d z \simeq \Delta z \sum_{k_{1} \leq k<k_{2}} \epsilon_{k}
$$

Here, $k_{n}$ is the index of the discrete vertical level that has its base at $z_{n}$. 
This vertically integrated entrainment rate also follows a gamma distribution, as shown below. In general, when we consider $n=1, \ldots, N$ realizations $x_{n}$ drawn from distribution $\Gamma(a, b)$ and define

$$
X=c \sum_{n=1}^{N} x_{n}
$$

where $c$ is a constant, then, owing to a special property of gamma distributions, the probability distribution of $X$ is given by $\Gamma(N a, b c)$. Hence, taking $\epsilon_{k}$ from distribution $\Gamma(\lambda \Delta z, \mu / \Delta z)$ implies that $\mathcal{E}_{z_{1}, z_{2}}$ given in Eq. (A1) is characterized by the distribution $\Gamma\left[\lambda\left(z_{2}-z_{1}\right), \mu\right]$. As such, the grid spacing no longer occurs as a parameter in the distribution. The standard deviation of the vertical entrainment rate between two levels $z_{1}$ and $z_{2}$ is derived from the standard deviation of $\mathcal{E}_{z_{1}, z_{2}}$ :

$$
\sigma_{\langle\epsilon\rangle}=\frac{\sigma_{\mathcal{E}_{z_{1}, z_{2}}}}{z_{2}-z_{1}}=\frac{\sqrt{\lambda} \mu}{\sqrt{z_{2}-z_{1}}} .
$$

To show why the vertically integrated entrainment rate is the key parameter in the parcel model, we consider the general solution to Eq. (7) for an entraining scalar $\psi$ :

$$
\frac{d}{d z} \psi_{p}=-\epsilon(z)\left(\psi_{p}-\psi_{e}\right)
$$

Along the parcel trajectory, the excess $\Delta \psi=\psi_{p}-\psi_{e}$ can be written as

$$
\Delta \psi\left(z_{2}\right)=\left\{\int_{z_{1}}^{z_{2}}\left(-\frac{d}{d \zeta_{1}} \psi_{e}\right) \exp \left[\int_{z 1}^{\zeta_{1}} \epsilon\left(\zeta_{2}\right) d \zeta_{2}\right] d \zeta_{1}+\Delta \psi\left(z_{1}\right)\right\} \times \exp \left[\int_{z 1}^{z 2}-\epsilon\left(\zeta_{1}\right) d \zeta_{1}\right] .
$$

The integrated entrainment rate appears in the integrand of the solution and, together with the environmental profiles and initial excess, determines the parcel properties.

\section{REFERENCES}

Betts, A. K., 1973: Non-precipitating cumulus convection and its parameterization. Quart. J. Roy. Meteor. Soc., 99, 178-196, doi:10.1002/qj.49709941915.

_- and B. A. Albrecht, 1987: Conserved variable analysis of the convective boundary layer thermodynamic structure over the tropical oceans. J. Atmos. Sci., 44, 83-99.

Blyth, A. M., and D. J. R. Cooper, 1988: Comparisons between observations of entrainment in Montana cumuli and results from a simple model. J. Atmos. Sci., 45, 1965-1969.

—_ W. A. Cooper, and J. B. Jensen, 1988: A study of the source of entrained air in Montana cumuli. J. Atmos. Sci., 45, 3944 3964.

Boatman, J. F., and A. H. Auer Jr., 1983: The role of cloud top entrainment in cumulus clouds. J. Atmos. Sci., 40, 1517-1534.

Böing, S. J., H. J. J. Jonker, A. P. Siebesma, and W. W. Grabowski, 2012: Influence of the subcloud layer on the development of a deep convective ensemble. J. Atmos. Sci., 69, 2682-2697.

Bryan, G. H., J. C. Wyngaard, and J. M. Fritsch, 2003: Resolution requirements for the simulation of deep moist convection. Mon. Wea. Rev., 131, 2394-2416.

Carpenter, R. L., Jr., K. K. Droegemeier, and A. M. Blyth, 1998: Entrainment and detrainment in numerically simulated cumulus congestus clouds. Part III: Parcel analysis. J. Atmos. Sci., 55, 3440-3455.

Dawe, J. T., and P. H. Austin, 2011: The influence of the cloud shell on tracer budget measurements of LES cloud entrainment. J. Atmos. Sci., 68, 2909-2920.

de Rooy, W. C., and Coauthors, 2012:Entrainment and detrainment in cumulus convection: an overview. Quart. J. Roy. Meteor. Soc., 139, 1-19, doi:10.1002/qj.1959.
Emanuel, K. A., 1994: Atmospheric Convection. Oxford University Press, $592 \mathrm{pp}$.

Grabowski, W. W., and H. Pawłowska, 1993: Entrainment and mixing in clouds: The Paluch mixing diagram revisited. J. Appl. Meteor., 32, 1767-1773.

— , and Coauthors, 2006: Daytime convective development over land: A model intercomparison based on LBA observations. Quart. J. Roy. Meteor. Soc., 132, 317-344, doi:10.1256/qj.04.147.

Heus, T., and H. J. J. Jonker, 2008: Subsiding shells around shallow cumulus clouds. J. Atmos. Sci., 65, 1003-1018.

— , G. van Dijk, H. J. J. Jonker, and H. E. A. Van den Akker, 2008: Mixing in shallow cumulus clouds studied by Lagrangian particle tracking. J. Atmos. Sci., 65, 2581-2597.

_ , and Coauthors, 2010: Formulation of and numerical studies with the Dutch Atmospheric Large-Eddy Simulation (DALES). Geosci. Model Dev., 3, 415-444.

Jensen, J. B., P. H. Austin, M. B. Baker, and A. M. Blyth, 1985: Turbulent mixing, spectral evolution and dynamics in a warm cumulus cloud. J. Atmos. Sci., 42, 173-192.

Jonas, P. R., 1990: Observations of cumulus cloud entrainment. Atmos. Res., 25, 105-127.

Khairoutdinov, M., and D. Randall, 2006: High-resolution simulation of shallow-to-deep convection transition over land. J. Atmos. Sci., 63, 3421-3436.

LaMontagne, R. G., and J. W. Telford, 1983: Cloud top mixing in small cumuli. J. Atmos. Sci., 40, 2148-2156.

Lin, C., and A. Arakawa, 1997a: The macroscopic entrainment processes of simulated cumulus ensemble. Part I: Entrainment sources. J. Atmos. Sci., 54, 1027-1043.

_, and _ 1997b: The macroscopic entrainment processes of simulated cumulus ensemble. Part II: Testing the entrainingplume model. J. Atmos. Sci., 54, 1044-1053.

Lu, C., Y. Liu, S. Niu, and A. M. Vogelmann, 2012: Lateral entrainment rate in shallow cumuli: Dependence on dry air sources and probability density functions. Geophys. Res. Lett., 39, L20812, doi:10.1029/2012GL053646.

Malinowski, S. P., and H. Pawłowska, 1989: On estimating the entrainment level in cumulus clouds. J. Atmos. Sci., 46, 2463-2465. 
Neggers, R. A. J., A. P. Siebesma, and H. J. J. Jonker, 2002: A multiparcel model for shallow cumulus convection. J. Atmos. Sci., 59, 1655-1668.

Nie, J., and Z. Kuang, 2012: Beyond bulk entrainment and detrainment rates: A new framework for diagnosing mixing in cumulus convection. Geophys. Res. Lett., 39, L21803, doi:10.1029/ 2012GL053992.

Paluch, I. R., 1979: The entrainment mechanism in Colorado cumuli. J. Atmos. Sci., 36, 2467-2478.

Raga, G. B., J. B. Jensen, and M. B. Baker, 1990: Characteristics of cumulus band clouds off the coast of Hawaii. J. Atmos. Sci., 47, 338-355.

Reuter, G. W., 1986: A historical review of cumulus entrainment studies. Bull. Amer. Meteor. Soc., 67, 151-154.

—_, and M. K. Yau, 1987: Mixing mechanisms in cumulus congestus clouds. Part I: Observations. J. Atmos. Sci., 44, 781-797.

Rodts, S. M. A., P. G. Duynkerke, and H. J. J. Jonker, 2003: Size distributions and dynamical properties of shallow cumulus clouds from aircraft observations and satellite data. J. Atmos. Sci., 60, 1895-1912.

Romps, D. M., and Z. Kuang, 2010: Nature versus nurture in shallow convection. J. Atmos. Sci., 67, 1655-1666.
Squires, P., 1958: The microstructure and colloidal stability of warm clouds, I. The relation between structure and stability. Tellus, 10, 256-261.

Stommel, H., 1947: Entrainment of air into a cumulus cloud. J. Meteor., 4, 91-94.

Sušelj, K., J. Teixeira, and D. Chung, 2013: A unified model for moist convective boundary layers based on a stochastic eddy-diffusivity/mass-flux parameterization. J. Atmos. Sci., 70, 1929-1953.

Taylor, G. R., and M. B. Baker, 1991: Entrainment and detrainment in cumulus clouds. J. Atmos. Sci., 48, 112-121.

Telford, J. W., 1975: Turbulence, entrainment, and mixing in cloud dynamics. Pure Appl. Geophys., 113, 1067-1084.

Verzijlbergh, R. A., H. J. J. Jonker, T. Heus, and J. Vilà-Guerau de Arellano, 2009: Turbulent dispersion in cloud-topped boundary layers. Atmos. Chem. Phys., 9, 1289-1302.

Wu, C., B. Stevens, and A. Arakawa, 2009: What controls the transition from shallow to deep convection? J. Atmos. Sci., 66 , 1793-1806.

Yeo, K., and D. M. Romps, 2013: Measurement of convective entrainment using Lagrangian particles. J. Atmos. Sci., 70, 266-277. 